Recebido em 11/2019. Aceito para publicação em 01/2020.

\title{
ANÁLISE DO COMPORTAMENTO TÉRMICO E REOLÓGICO DO ABS APÓS EXTRUSÃO
}

\section{ANALYSIS OF ABS THERMAL AND RHEOLOGICAL BEHAVIOR AFTER EXTRUSION}

\author{
Paulo Estevão Quimas Quelho' \\ Rafael de Aquino Alvarenga ${ }^{2}$ \\ Pamela Nunes Meireles ${ }^{3}$ \\ Alexandre Alvarenga Palmeira ${ }^{4}$ \\ Sérgio Roberto Montoro ${ }^{5}$ \\ Cirlene Fourquet Bandeira ${ }^{6}$
}

Resumo: Atualmente, vários materiais são extrudados, entre eles o ABS (Acrilonitrila Butadieno Estireno), que é um termoplástico com grande aplicabilidade em vários setores da indústria, principalmente automotiva. Entretanto, as etapas de aquecimentos do processo, bem como o tempo de exposição podem causar degradação do polímero. Estes defeitos podem gerar uma série de inconvenientes, que vão desde a perda da peça, até o risco de acidentes devido aos pontos de propagação de falhas. Em decorrência disto, são necessárias análises que comprovem a variação das características do material em função da temperatura, especialmente se o equipamento utilizado para o processo de extrusão for experimental. As análises que apresentam baixo custo e rapidez e que determinam a temperatura de degradação ou a diminuição do tamanho da molécula por quebra da mesma são feitas via TGA (Análise Termogravimétrica) ou pelo índice de fluidez. Sendo assim, o presente trabalho desenvolveu um estudo do comportamento de 3 diferentes tipos de ABS (acrilonitrila-butadieno-estireno) denominados AE800, de AG1000 MG94, extrudados a uma rotação de 60RPM, para avaliar o comportamento termo cinético por meio de ensaios de TGA e índice de fluidez. Os resultados demonstram que em alguns casos, o índice de fluidez se mostra mais eficaz que a termogravimetria para determinar esse parâmetro e que apesar das pequenas variações nas propriedades do ABS, é possível sua utilização.

Palavras-chave: Termogravimetria; índice de fluidez; extrusora experimental.

Abstract: Currently, various materials are extruded, among them ABS (Acrylonitrile Butadiene Styrene) which is a thermoplastic with great applicability in various industrial sectors, especially the automotive. However, the process heating steps, as well as exposure time, may cause polymer degradation. These defects can create a number of inconveniences, ranging from piece loss to the risk of accidents due to fault propagation points. As a result, analyses are needed to prove the variation of the characteristics of the material as a function of temperature, especially if the equipment used for the extrusion process is experimental. The analyses that present low cost, speed, and which determine the temperature of degradation or the size reduction of the molecule by breaking it are based on the temperatures of degradation es, are performed via TGA (Thermogravimetric Analysis) and flow rate. Thus, the present work developed a study of the behavior of 3 different types of ABS (acrylonitrile butadiene styrene) called AE800 and AG1000 MG94, when extruded at 60RPM, to evaluate the thermo-kinetic behavior by TGA and flow rate tests. The results demonstrate that in some cases, the flow rate tests are more effective than

\footnotetext{
1 Graduado, aluno do mestrado, UniFOA, Brasil. E-mail: pauloquimas@gmail.com.

2 Graduado, aluno do mestrado, UniFOA, Brasil. E-mail: rafael_alvarenga_@hotmail.com.

3 Graduada, aluna da Eng. Ambiental, UniFOA, Brasil. E-mail: pamela.meireles1@gmail.com.

4 Doutor, professor, UniFOA, Brasil. E-mail: alexandre.palmeira@foa.org.br.

5 Doutor, professor, UniFOA, Brasil. E-mail: E-mail: sergio.montoro@foa.org.br.

6 Doutora, professora, UniFOA e UNESP-FEG, Brasil. E-mail: cirlenefourquet@yahoo.com.br.
} 
thermogravimetry to determine this parameter and that despite the slight variations in ABS properties, its use is possible.

Keywords: Thermogravimetry; flow rate; experimental extruder.

\section{INTRODUÇÂO}

A extrusão se caracteriza por ser um processo semicontínuo para produção de peças. De forma geral, os materiais extrudados passam por três zonas denominadas de alimentação, compressão e dosagem até serem forçados através de uma matriz, adquirindo uma forma pré-determinada (MARTINS, 2014; YANG, 2018).

Neste processo, os materiais termoplásticos são introduzidos na extrusora por meio de um funil, caindo por ação da gravidade em uma rosca contida num cilindro aquecido numa temperatura próximo a temperatura de fusão do polímero. $\mathrm{Na}$ sequência, o material é comprimido e sofre plastificação, sendo, transportados, até a etapa seguinte que garante a homogeneização e a vazão da mistura, através da pressão gerada, para a matriz (MARTINS, 2014; CHOE et al., 2014).

Este processo de conformação surgiu em meados 1797, mas sua aplicabilidade cresceu de forma vertiginosa a partir da segunda guerra mundial, principalmente, por apresentar vantagens, tais como a obtenção de peças livres de trincas, controle dimensional elevado, possibilidade de produção de volumes variados e produção de peças com os mais variados designs (QUELHO, 2018).

Atualmente, vários materiais são extrudados, entre eles o $A B S$ (AcrilonitrilaButadieno-Estireno) que é um termoplástico com grande aplicabilidade em vários setores da indústria, principalmente automotiva, na produção de painéis, ou como filamentos em impressoras 3D. Entretanto, as etapas de aquecimentos do processo, bem como o tempo de exposição, quando não bem dimensionadas, podem causar degradação do polímero extrudado. Estes problemas podem se manifestar na forma de estrias marrons, pontos pretos, carbonização, estrias prateadas ou até não ser visível (DE BLASIO, 2007; MARTINS, 2014; ROCHA et al., 2014).

Estes defeitos podem gerar uma série de inconvenientes, que vão desde o aumento do custo devido à perda da peça, até o risco de acidentes devido aos pontos de propagação de falhas criados por estes materiais. Mediante esta situação, são necessárias análises que comprovem a variação das características do material em função da temperatura, especialmente se o equipamento utilizado para o processo de extrusão for experimental (DE BLASIO, 2007; QUELHO, 2018).

A análise mais comum, com um baixo custo e rapidez que determinam a temperatura de degradação ou a diminuição do tamanho da molécula por quebra se baseiam nas temperaturas degradação, que é avaliada em TGA (Análise Termogravimétrica). Além disto as análises reométricas também são muito utilizadas como suporte para determinar processabilidade e desenvolvimento de novos produtos 
(BANDEIRA, 2015; QUELHO, 2018; YANG, 2018).

Assim, essa pesquisa tem o objetivo de avaliar o comportamento reológico e térmico de três diferentes tipos de $A B S$, quando submetidos a um processo de extrusão em uma extrusora experimental à $60 \mathrm{RPM}$ e temperaturas de controle de $220^{\circ} \mathrm{C}$ para $\mathrm{T} 1,225^{\circ} \mathrm{C}$ para $\mathrm{T} 2 / \mathrm{T} 3$ e $240^{\circ} \mathrm{C}$ para $\mathrm{T} 4$ e verificar se os materiais mantêm suas características através de ensaios de TGA e índice de Fluidez.

\section{REVISÃO DA LITERATURA}

\subsection{Polímeros}

Os materiais poliméricos vêm ganhando importância, sobretudo nas últimas décadas, com usos que vão desde utensílios domésticos, até aplicações em equipamentos automotivos, aeronáuticos, de construção civil, entre outros (CAMPBELL; PETHRICK; WHITE, 2000; CANEVAROLO JUNIOR, 2002; QUELHO, 2018; YANG, 2018).

Os polímeros são produzidos, geralmente, a partir do petróleo e são definidos como compostos de origem natural ou sintética, formados pela repetição de um grande número de unidades químicas denominadas de meros. Eles são classificados de acordo com seu comportamento mecânico como plásticos, borrachas e fibras.

Os plásticos possuem alta massa molecular, e encontra-se em estado sólido na forma de produto acabado e podem ser subdivididos em termoplásticos que se caracterizam por apresentarem capacidade de amolecer e fluir quando expostos a um aumento de temperatura e/ou pressão e que retornam à condição inicial quando é retirada a solicitação, os termorrígidos que apresentam uma transformação química irreversível quando os monômeros são expostos ao aquecimento, tornando-se rígidos devido a formação de ligações cruzadas entre as cadeias e que degradam com um segundo aquecimento devido ao nível de energia elevado necessário para que haja quebra dessas ligações e os elastômeros, também conhecidos como borrachas, que sofrem cura com o primeiro aquecimento e com a continuidade do aumento de temperatura/tempo de aquecimento, eles se degradam (QUELHO, 2018; YANG, 2018).

Estes materiais apresentam grande importância econômica com uma produção de 322 milhões de toneladas em 2015, com perspectivas de dobrarem de volume nos próximos 20 anos. Dentre estes materiais, o ABS se destaca, representando $14 \%$ da produção mundial de plásticos (COMISSÃO EUROPÉIA, 2018; CORDOBELLO, 2003).

\subsection{ABS}

O ABS é um termoplástico derivado do petróleo cuja composição é formada por acrilonitra-butadieno-estireno, nas proporções de $15 \%$ a $30 \%, 5 \%$ a $15 \%$ e $40 \%$ a $60 \%$ respectivamente. Desta forma é possível produzir vários tipos diferentes de ABS que 
podem ser aplicados nos mais variados segmentos da indústria, com produção anual é de aproximadamente 3 milhões de toneladas (CHOE et al., 2014, QUELHO, 2018).

Sua produção ocorre, industrialmente, pela polimerização do butadieno que se transforma em polibutadieno e a transformação do estireno e da acrilonitrila no copolímero denominado SAM (copolímero de estireno-acrilonitrila) que posteriormente são misturados de modo a formar o ABS (QUELHO, 2018).

Este copolímero apresenta grande aplicabilidade na indústria automotiva, embalagens, móveis, fios cabos e em outros seguimentos visto que apresenta preço relativamente baixo, baixa densidade e resistência ao impacto, à abrasão, à temperaturas (suportam temperaturas entre $-20^{\circ} \mathrm{C}$ a $80^{\circ} \mathrm{C}$ ) e à tração, além de apresentar fácil moldabilidade tanto por injeção quanto por extrusão, com o mínimo de deformação e ser isolantes elétricos (LI; SHIMIZU, 2009; MAIS POLÍMEROS, 2019; ROCHA et al., 2014).

Em temperatura ambiente é inerte, não apresenta risco à saúde e apresenta excelente aspecto visual, podendo ser metalizado e pigmentado com ótimos resultados (QUELHO, 2018).

\subsection{Processamento de Materiais Poliméricos}

Os principais métodos industriais para processamento de materiais poliméricos, são os processos de extrusão, de injeção, de sopro e de calandragem, sendo que para os materiais termoplásticos, o processo de extrusão é considerado um dos mais importantes (QUELHO, 2018).

O processo de extrusão é comumente utilizado no processamento de elementos estruturais de plástico reciclado. Nesse processo os grãos (pellets) são submetidos a zonas de calor, a fim de fluidificá-los e posteriormente moldá-los. Desta forma, este processo, tem por objetivo a conversão de um material sólido em um fluído através da adição de calor a sua estrutura física, e aplicação de trabalho, submetendo este material a pressões. O esforço mecânico sobre o material é realizado por um parafuso, que ao realizar o movimento rotativo, empurra o material, na direção de uma matriz, tal qual o transforma em uma massa uniforme (GALDÁMEZ, 2002; QUELHO, 2018; YANG, 2018).

Entretanto, o aquecimento que ocorre durante o processo pode acarretar a degradação do polímero que irá apresentar defeitos que vão desde o aumento do custo devido à perda da peça, até o risco de acidentes por pontos propagação de falhas criados por estes materiais (CHEN et al., 2019; MARTINS, 2014).

Estes problemas podem se manifestar na forma de estrias marrons, pontos pretos, carbonização, estrias prateadas ou até não ser visível (DE BLASIO, 2007; MARTINS, 2014). 


\section{MATERIAIS E MÉTODOS}

\subsection{Materiais}

Foram selecionados três ABS diferentes para a realização deste trabalho. Sendo:

I. ABS AG1000 fabricado pela Videolar-Innova S/A e adquiridos no mercado de São Paulo. Este material é considerado de altíssima resistência ao impacto;

II. ABS AE8000 fabricado pela Videolar-Innova S/A e foi adquirido no mesmo fornecedor anterior. Este tipo de ABS é considerado de alto impacto e de elevada rigidez;

III. ABS MG94 fabricado pela Cycolac ${ }^{\mathrm{TM}}$ e adquirido em São Paulo. Este termoplástico apresenta como principal característica sua resistência ao impacto.

\subsection{Métodos}

\subsubsection{Preparação dos Corpos de Prova via Extrusão}

Os compósitos poliméricos foram produzidos via extrusão, em uma extrusora de rosca simples com o fuso de aço inoxidável que foi produzida pelo aluno do Mestrado Profissional de Materiais (MeMat) do UniFOA na Dissertação "Desenvolvimento de Extrusora Experimental e Software para Controle e Supervisão das Variáveis de Extrusão do ABS".

Esta extrusora é composta de três zonas distintas denominadas de zona de alimentação, zona de compressão e zona de regulação. A alimentação do processo foi feita por adição do material pelo alimentador e o controle de temperatura foi feito via digital através de controle proporcional integral derivativo (PID) das zonas de aquecimento $\mathrm{T} 1\left(220^{\circ} \mathrm{C}\right), \mathrm{T} 2\left(225^{\circ} \mathrm{C}\right), \mathrm{T} 3\left(230^{\circ} \mathrm{C}\right)$ e $\mathrm{T} 4\left(240^{\circ} \mathrm{C}\right)$ e a velocidade da rosca foi de 60 rotações por minuto (rpm).

\subsection{2 Índice de Fluidez}

Os ensaios de índice de fluidez, foram realizados no equipamento NZ Philpolymer, modelo XRL - 400 localizado no Laboratório Afinko em São Carlos.

As condições de teste foram baseadas na norma ISO 1133:2011 que determina a temperatura do laboratório de $23^{\circ} \mathrm{C}$ e umidade de $50 \%$, carga aplicada de $10,0 \mathrm{~kg}$, tempo de pré-aquecimento de $7 \mathrm{~min}$, temperatura de ensaio de $220^{\circ} \mathrm{C}$, tempo de corte de 10 s e número mínimo de medições igual a 5.

Foram realizadas 5 amostras de cada material extrudado sob condições de rotação de 60 RPM e 5 amostras de cada material virgem (sem passar pelo processo de extrusão) para verificar se o processo havia modificado as características do material. 


\subsubsection{Termogravimetria}

Os ensaios de análise térmica foram realizados no laboratório de análise térmica do Centro Universitário de Volta Redonda e analisados de acordo com a ASTM E2550:2017. O equipamento utilizado foi o PerkinEImer modelo STA6000 sob as seguintes condições: faixa de trabalho de temperatura ambiente $(25 \mathrm{oC})$ até $950^{\circ} \mathrm{C} \mathrm{com}$ taxa de aquecimento de $10^{\circ} \mathrm{C} / \mathrm{min}$, fluxo de nitrogênio de $20 \mathrm{~mL} / \mathrm{min}$, panela de alumina e massa aproximada de $10 \mathrm{mg}$.

Foram realizadas amostras de materiais extrudados sob condições de rotação de 60 RPM e nas amostras, de cada material virgem, para verificar se o processo havia modificado as temperaturas de início de temperatura e o percentual de perda de cada material.

\section{RESULATADOS E DISCUSSÕES}

\subsection{Ensaios Reológicos}

\subsubsection{ABS AE8000}

Ocorreu o aumento da fluidez e, consequentemente, a redução da viscosidade devido à cisão de cadeias moleculares do material em decorrência do aquecimento durante o processo de extrusão.

Pode-se observar (Figura 1) que os materiais virgens apresentaram índice de fluidez de 5,2 com coeficiente de variação de 1,1\%, enquanto os extrudados apresentaram índice de fluidez de 6,2 e coeficiente de variação de aproximadamente $0,7 \%$, demonstrando que as amostras são homogêneas. Além disto, as amostras extrudadas, em relação às amostras virgens apresentaram uma variação do índice de fluidez de aproximadamente $20 \%$, fator relevante no total.

Figura 1 - Resultados do índice fluidez do ABS AE800.

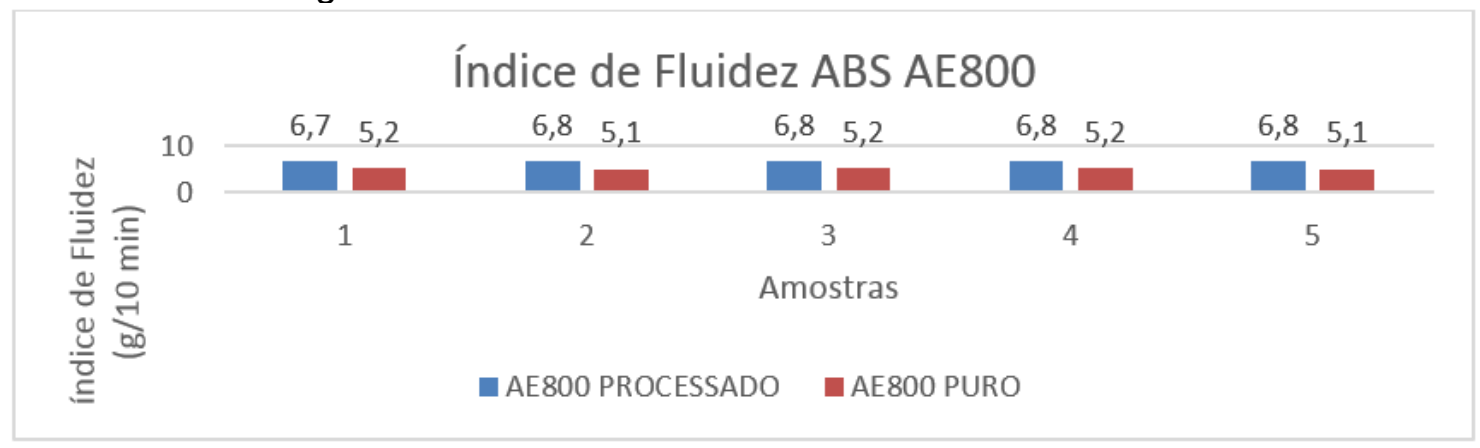

Fonte: Os autores.

\subsubsection{ABS MG94}

Ocorreu um comportamento inverso ao da amostra anterior (ABS AE8000), com 
diminuição do índice de fluidez dos materiais extrudados em $5 \%$ em relação aos materiais virgens (Figura 2).

Apesar disto, os resultados do índice de fluidez (IMF) mostram que o material extrudado apresenta, em média, um IMF de 32,84 $\pm 0,05477 \mathrm{~g} / 10$ min e o material puro $34,28 \pm 0,29495 \mathrm{~g} / 10 \mathrm{~min}$. Esta pequena diferença demonstra que o material processado sob as condições térmicas de $220^{\circ} \mathrm{C}(\mathrm{T} 1), 225^{\circ} \mathrm{C}(\mathrm{T} 2), 230^{\circ} \mathrm{C}(\mathrm{T} 3)$ e $240^{\circ} \mathrm{C}$ (T4), a uma velocidade de rosca de 60 RPM, não sofreu alterações significativas e quebra das cadeias, uma vez que a rosca desempenhou a função apenas de transporte e compactação do material que teve, provavelmente, sua densidade aumentada, influenciando os valores de IMF.

Figura 2 - Resultados do índice fluidez do ABS MG94.

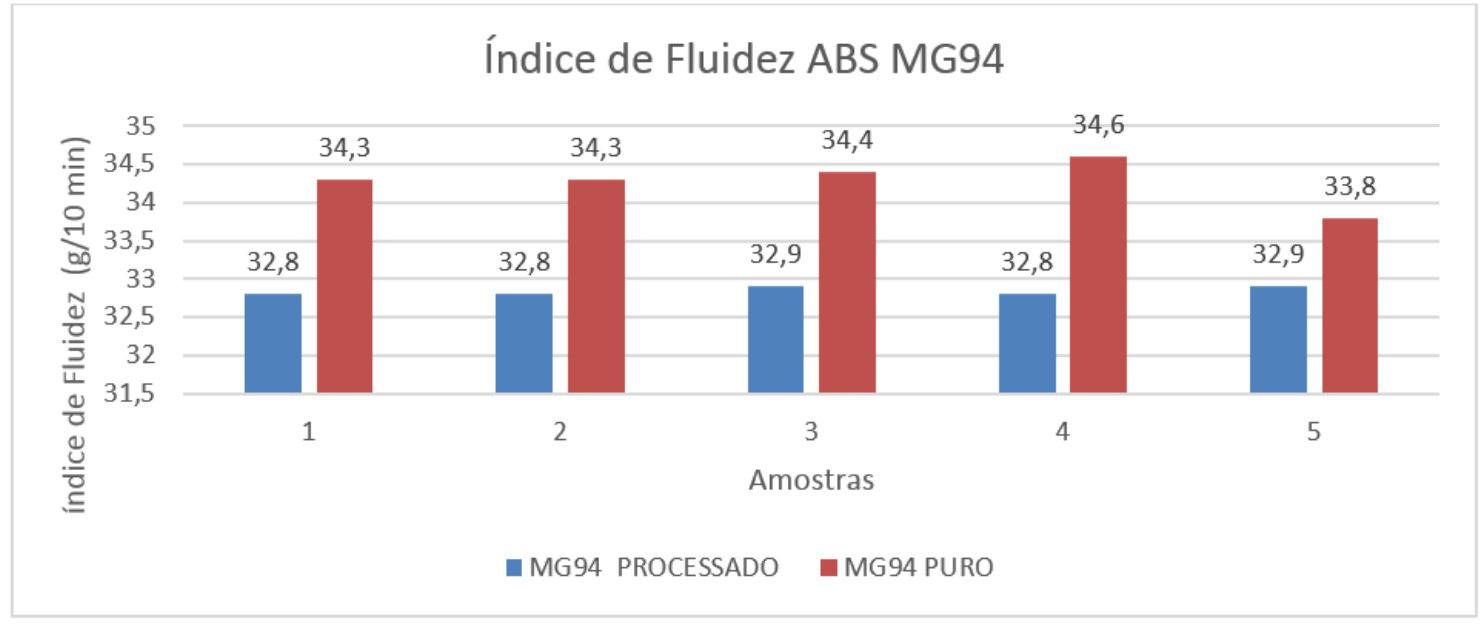

Fonte: Os autores.

\subsubsection{ABS AG100}

Não houve, conforme observado no resultado do índice de fluidez (Figura 3) uma variação significativa dos resultados. O material extrudado apresenta um IMF médio de $25,24 \pm 0,0894 \mathrm{~g} / 10 \mathrm{~min}$ e o material puro $25,42 \pm 0,13038 \mathrm{~g} / 10 \mathrm{~min}$. Conforme observado por Gomes (1985), afirma-se que quanto menor for o CV, mais homogêneos são os dados estudados. Portanto, o coeficiente de variação da amostra processada sendo 0,35\% da média, permite concluir que as amostras são homogêneas em função da baixa dispersão. Logo, com este resultado ficam garantidas as propriedades reológicas do material. 
Figura 3 - Resultados do índice fluidez do ABS AG100.

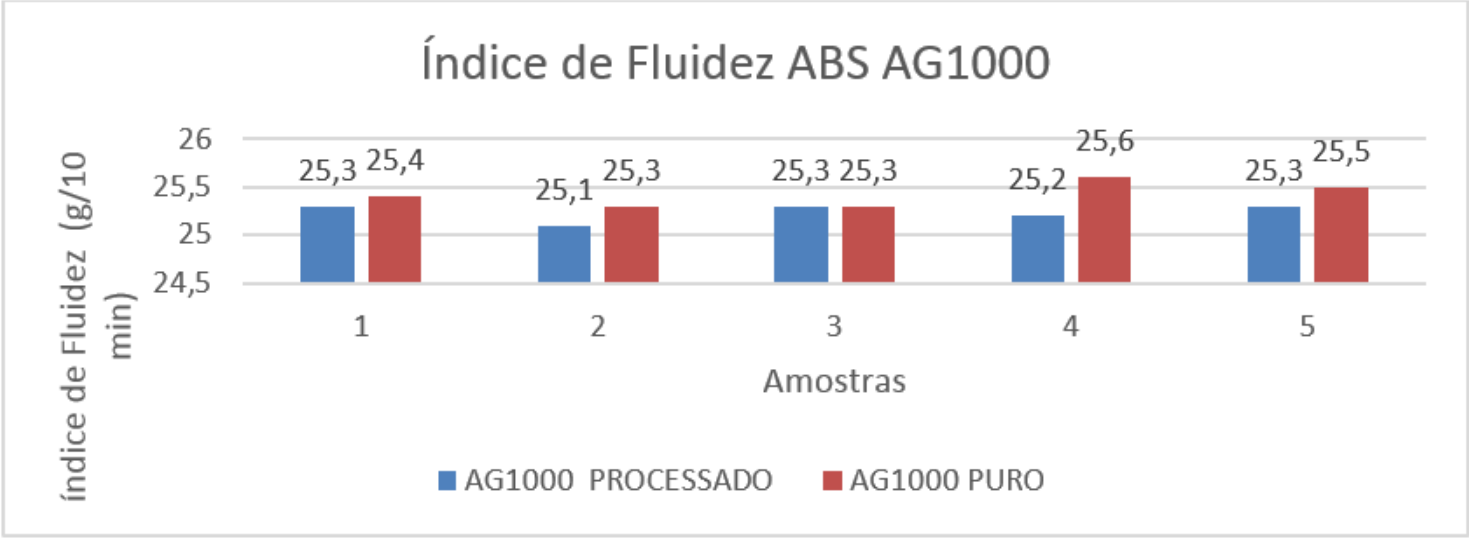

Fonte: Os autores.

\subsection{Ensaios Termogravimétricos}

\subsubsection{ABS AE8000}

Tanto o ABS virgem quanto o processado (Figuras 4 e 5), apresentam temperaturas extrapoladas de início de degradação (Tonset) em torno de $412^{\circ} \mathrm{C}$, demostrando que o processamento do material não afetou, de forma significativa, esta propriedade do polímero.

O mesmo contexto pode ser observado para a temperatura de final de degradação (Tendset) (Tabela 1), que compara o resultado do ABS P (puro) com o ABS V (virgem). As variáveis de perda de massa e resíduo se comportaram de maneira similar com valores constantes próximos à $97,5 \%$ e $2 \%$ respectivamente.

Figura 4 - Curva termogravimétrica do ABS AE8000 virgem.

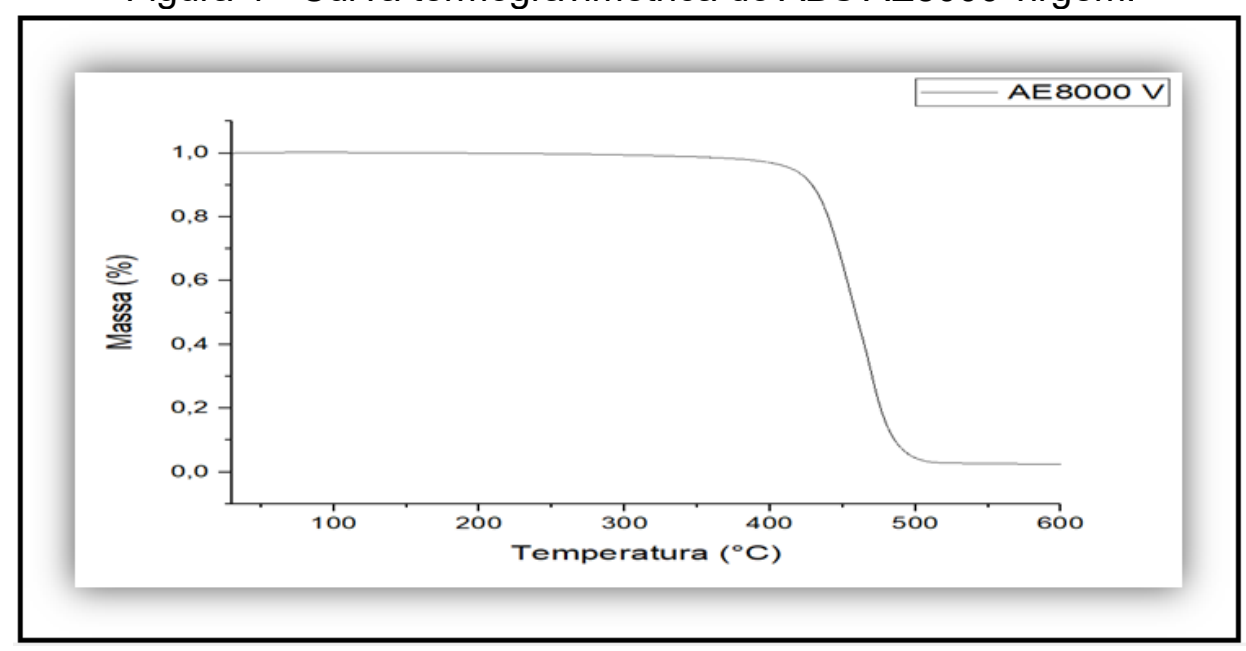

Fonte: Os autores. 
Figura 5 - Curva termogravimétrica do ABS AE8000 processado.

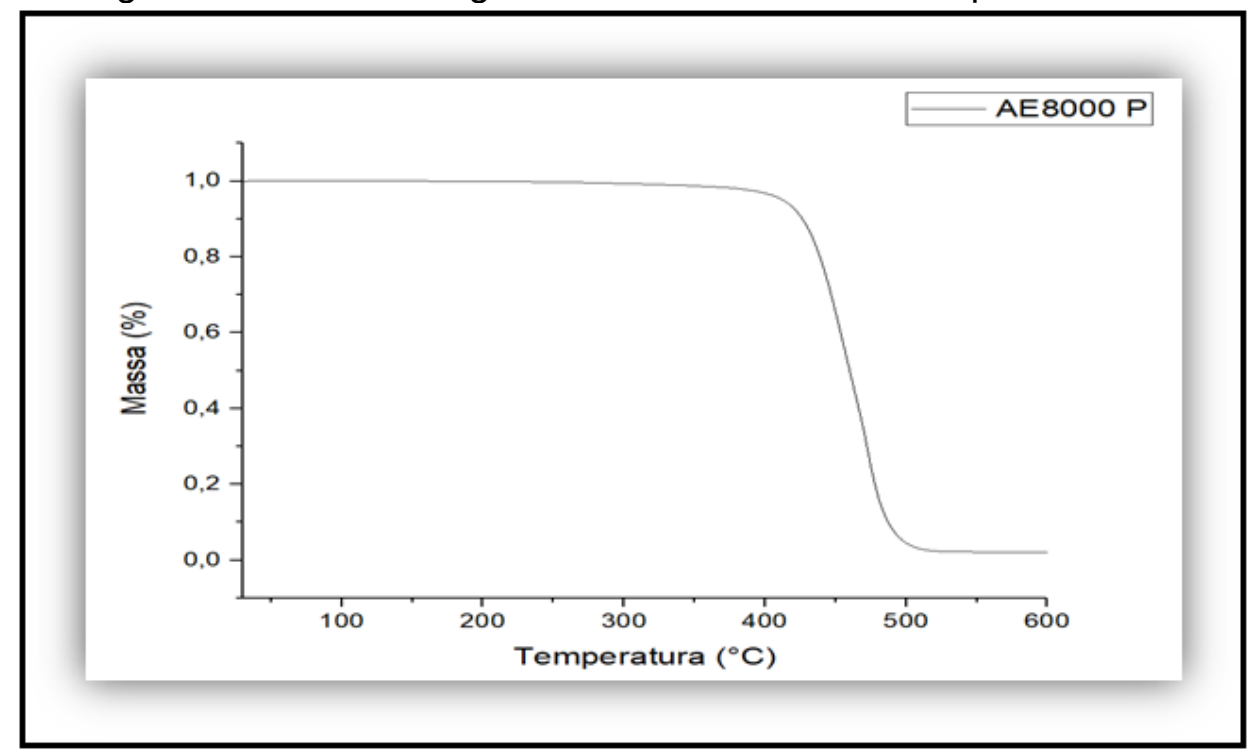

Fonte: Os autores.

Tabela 1 - Valores obtidos na curva de termogravimetria do ABS AE8000.

\begin{tabular}{ccc}
\hline & \multicolumn{2}{c}{ ABS AE8000 } \\
\cline { 2 - 3 } & Processado & Virgem \\
\hline Tonset $\left({ }^{\circ} \mathrm{C}\right)$ & 411,74 & 411,54 \\
$\begin{array}{c}\text { Tendset } \\
\left({ }^{\circ} \mathrm{C}\right)\end{array}$ & 466,91 & 465,15 \\
$\begin{array}{c}\text { Perda de } \\
\text { massa } \\
\text { entre } 30 \text { e } \\
550^{\circ} \mathrm{C}(\%) \\
\text { Resíduo } \\
(\%)\end{array}$ & 97,86 & 97,42 \\
\hline \multicolumn{3}{c}{ Fonte: Os autores. }
\end{tabular}

\subsubsection{ABS AG100}

Os gráficos da análise térmica do AG1000 V (Figura 6) e AG1000 P (Figura 7) geraram os resultados disponíveis na Tabela 2.

Os materiais apresentaram temperaturas, de início, extrapoladas de degradações próximas, uma temperatura de endset de aproximadamente $465^{\circ} \mathrm{C}$ para as duas análises e perda de massa, observada no intervalo de 30 a $550^{\circ} \mathrm{C}$, de $99 \%$ para ambos os polímeros, o que permite afirmar que o comportamento térmico do material não foi alterado após o processamento. 
Figura 6 - Curva termogravimétrica do ABS AG100 virgem.

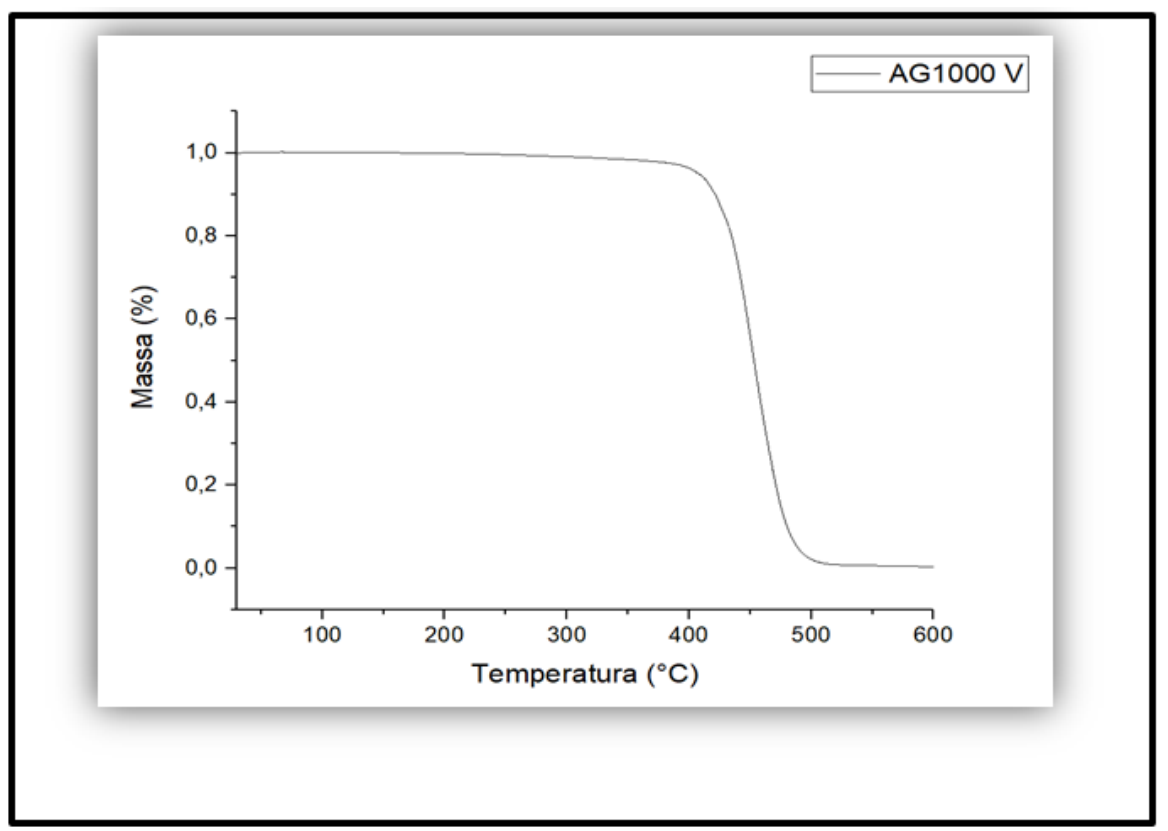

Fonte: Os autores.

Figura 7 - Curva termogravimétrica do ABS AG100 processado.

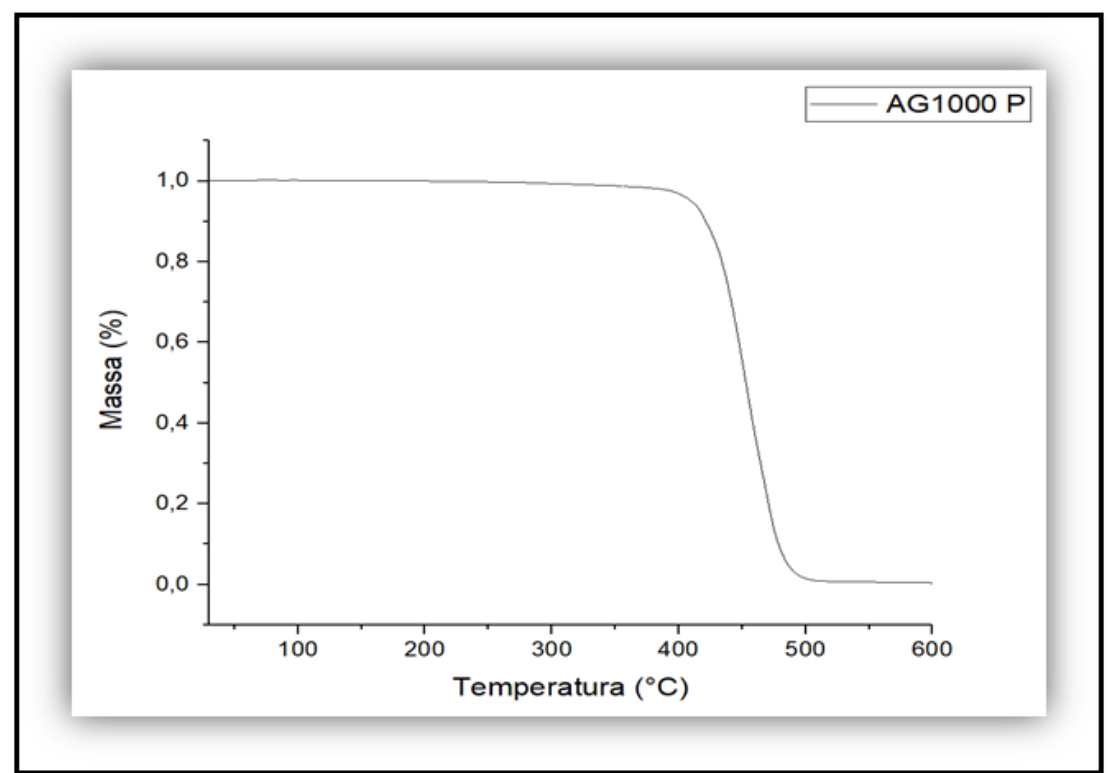

Fonte: Os autores. 
Tabela 2 - Valores obtidos na curva de termogravimetria do ABS AG100.

\begin{tabular}{|c|c|c|}
\hline & \multicolumn{2}{|c|}{ ABS AG100 } \\
\hline & Processado & Virgem \\
\hline Tonset $\left({ }^{\circ} \mathrm{C}\right)$ & 409,02 & 407,78 \\
\hline $\begin{array}{c}\text { Tendset } \\
\left({ }^{\circ} \mathrm{C}\right)\end{array}$ & 465,29 & 465,52 \\
\hline $\begin{array}{l}\text { Perda de } \\
\text { massa } \\
\text { entre } 30 \mathrm{e} \\
550^{\circ} \mathrm{C}(\%)\end{array}$ & 99,35 & 99,54 \\
\hline $\begin{array}{l}\text { Resíduo } \\
(\%)\end{array}$ & 2,00 & 2,00 \\
\hline
\end{tabular}

\subsubsection{ABS MG94}

Similar aos dois últimos materiais analisados (EA8000 e AG100), o comportamento térmico do MG94 está representado graficamente (Figuras 8 e 9). Os resultados das análises demonstram que não há mudanças térmicas significativas que alterassem as propriedades térmicas esperadas para o material (Tabela 3). Texto com fonte diferente dos demais.

Figura 8 - Curva termogravimétrica do ABS MG94 virgem.

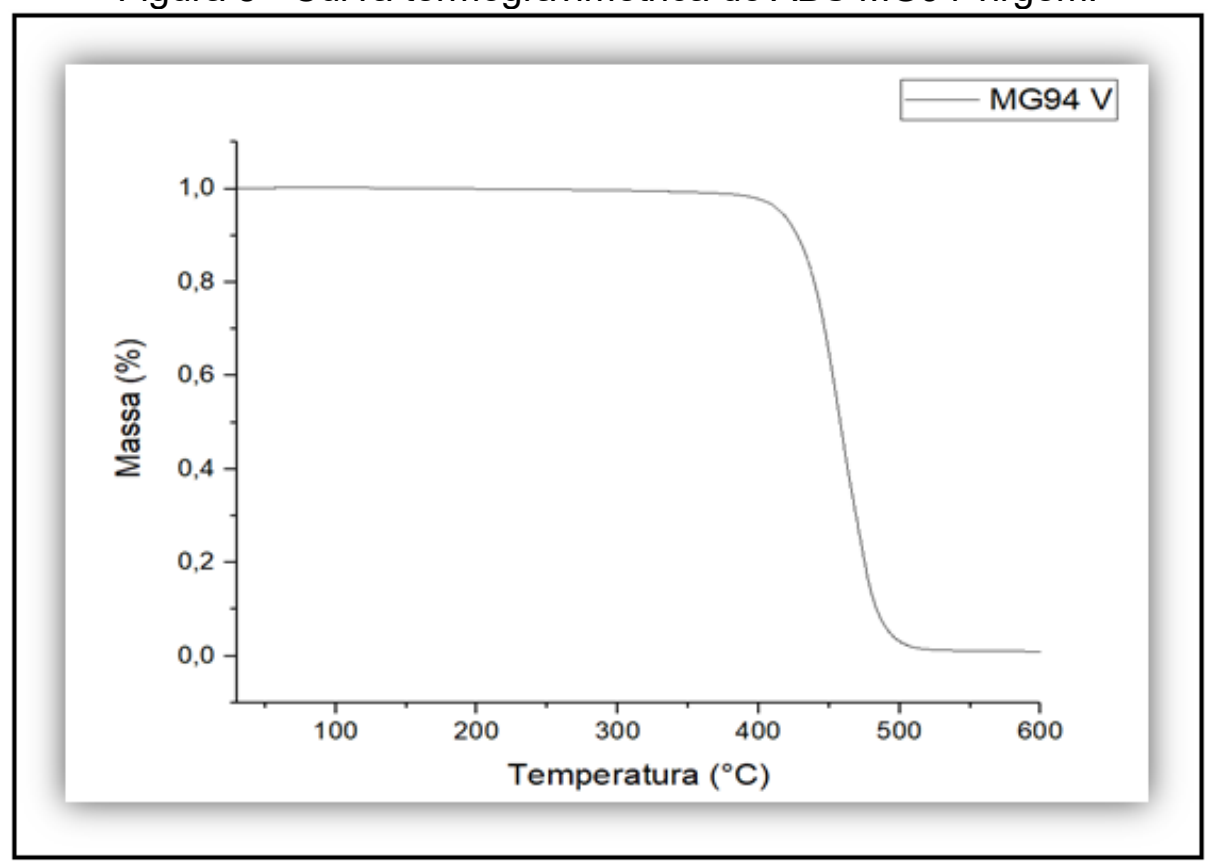

Fonte: Os autores. 
Figura 7 - Curva termogravimétrica do ABS MG94 processado.

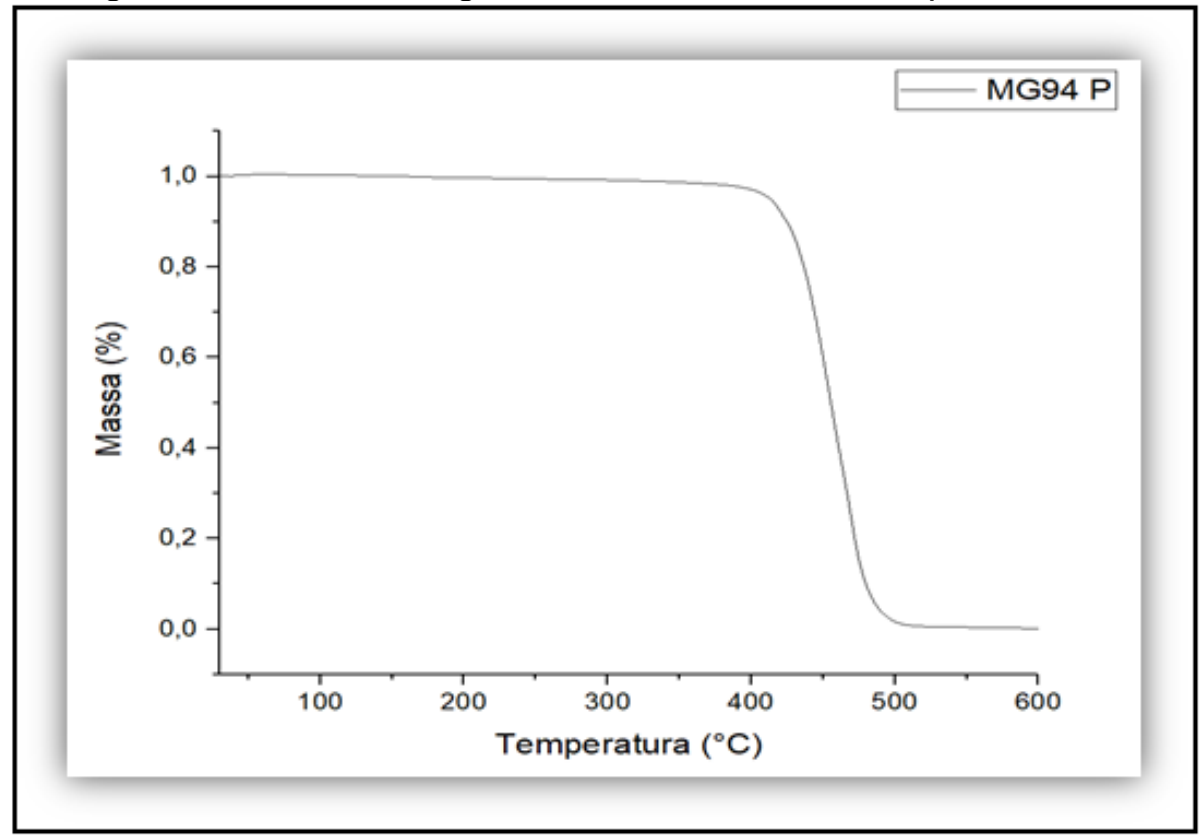

Fonte: Os autores.

Tabela 3 - Valores obtidos na curva de termogravimetria do ABS MG94.

\begin{tabular}{ccc} 
& \multicolumn{2}{c}{ ABS MG94 } \\
\cline { 2 - 3 } & Processado & Virgem \\
\hline Tonset $\left({ }^{\circ} \mathrm{C}\right)$ & 410,23 & 412,35 \\
$\begin{array}{c}\text { Tendset } \\
\left({ }^{\circ} \mathrm{C}\right)\end{array}$ & 466,34 & 468,22 \\
$\begin{array}{c}\text { Perda de } \\
\text { massa } \\
\text { entre } 30 \text { e } \\
550^{\circ} \mathrm{C}(\%) \\
\text { Resíduo } \\
(\%)\end{array}$ & 99,85 & 99,00 \\
\hline
\end{tabular}

Fonte: Os autores.

\section{CONCLUSÕES}

A partir das análises para determinação do índice de fluidez, foi possível observar que dois materiais, o MG94 e o AG1000, apresentaram comportamentos próximos com diminuição do IMF, comportamentos estes que ocorreram, provavelmente, devido ao LD (comprimento) da rosca ser pequeno, o que não proporcionou um tempo adequado de trabalho, fazendo com que apenas houvesse o transporte e compactação da amostra. Já a amostra AE800 apresentou cisão de cadeias moleculares do material, com aumento de $20 \%$ do IMF, em decorrência do aquecimento durante o processo de extrusão.

O comportamento térmico, entretanto, para os três polímeros estudados, mostra que não houve mudanças significativas devido ao processamento em extrusora. 
Desta forma, pode-se notar que os três materiais, sofreram pequenas variações nas propriedades do polímero, que, no entanto, não inviabiliza seu uso em aplicações de forma geral.

\section{AGRADECIMENTOS}

Ao UniFOA que colaborou com o andamento deste trabalho.

\section{REFERÊNCIAS}

ASTM E2550 - AMERICAN SOCIETY FOR TESTING AND MATERIALS. Standard Test Method for Thermal Stability by Thermogravimetry. United States: s.n., 2017.

BANDEIRA, C. F. Obtenção e caracterização de compósitos de benzoxazina/fibra de carbono. 2015. Dissertação (Doutorado na área de Materiais) - Universidade Estadual Paulista, Guaratinguetá, 2015.

CAMPBELL, D.; PETHRICK, R. A.; WHITE, J. R. Polymer Characterization: physical techniques. Boca Raton: CRC Press - Taylor \& Francis Group, 2000.

CANEVAROLO JUNIOR, S. V. Técnicas de Caracterização de Polímeros. São Paulo: Artliber, v. 1, 2004.

CHEN, F. et al. Fabrication of novel resinous diamond composites with acrylonitrile butadiene styrene/polyvinyl chloride/dioctyl phthalate/diamond by hot pressing molding. Journal of Materials Research, Cambridge, v. 34, n.10, 2019.

$\mathrm{CHOE}, \mathrm{I}$. J. et al. Mechanical properties of acrylonitrile-butadiene-styrene copolymer/poly (I-lactic acid) blends and their composites. Journal of Applied Polymer Science, Hoboken, v. 131, n.11, 2014.

COMISSÃO EUROPÉIA. Comunicação da Comissão ao parlamento europeu, ao conselho, ao comité econômico e social europeu e ao comité das regiões. Uma Estratégia Europeia para os Plásticos na Economia Circular. Estrasburgo, 2018.

CORDOBELLO, F. S. Polímeros do Futuro: tendências e oportunidades: palestras técnicas (II). Polímeros Journal, v. 13, n. 1, 2003.

DE BLASIO, C. A. Solução de Defeitos na Moldagem por Injeção de Termoplásticos. 2007. Dissertação (Mestrado acadêmico) - Universidade Estadual de Campinas, Campinas, 2007.

GALDÁMEZ, E. V. C. Aplicação das técnicas de planejamento e análise de experimentos na melhoria da qualidade de um processo de fabricação de produtos plásticos. 2002. Dissertação (Mestrado Profissional em Materiais) Universidade de São Paulo, São Carlos, 2002.

GOMES, P. Curso de estatística experimental. ESALQ/USP. Piracicaba-SP: s.n., 1985. 
ISO 1133. INTERNATIONAL ORGANIZATION FOR STANDARDIZATION. Plastics Determination of the melt mass-flow rate (MFR) and melt volume-flow rate (MVR) of thermoplastics. United States: s.n., 2011.

LI, Y.; SHIMIZU, H. Improvement in toughness of poly(I-lactide) (PLLA) through reactive blending with acrylonitrile-butadiene-styrene copolymer (ABS): Morphology and properties. European Polymer Journal, Oxford, v. 45, n. 3, 2009.

MAIS POLÍMEROS. Plastico ABS e suas principais características e aplicações. Disponível em: http://www.maispolimeros.com.br/2018/09/24/plastico-abs-e-suas-princi pais-caracteristicas-e-aplicacoes/. Acesso em: 15 out. 2019.

MARTINS, C. A. Estudo de comportamento de defeitos de superfície da matériaprima em operações de extrusão a frio de aços baixo carbono. 2014. Dissertação (Mestrado em Engenharia Mecânica) - Universidade Federal de São João del-Rei, São João del-Rei, 2014.

QUELHO, P. E. Q. Desenvolvimento de extrusora experimental e software para controle e supervisão das variáveis de extrusão do ABD. 2018. Dissertação (Mestrado Profissional em Materiais) - Centro Universitário de Volta Redonda UniFOA, Volta Redonda, 2018.

ROCHA, C. R. et al. Novel ABS-based binary and ternary polymer blends for material extrusion 3D printing. Journal of Materials Research, Cambridge, v. 29, n. 17, 2014.

YANG, R. Analytical methods for polymer characterization. New York: CRC Press, 2018. 